\title{
MID-IR OBSERVATIONS AND A REVISED TIME DELAY FOR THE GRAVITATIONAL LENS SYSTEM QUASAR HE 1104-1805
}

\author{
Shawn Poindexter, ${ }^{1}$ Nicholas Morgan, ${ }^{1}$ Christopher S. Kochanek, ${ }^{1}$ and Emilio E. Falco ${ }^{2}$ \\ Received 2006 December 1; accepted 2007 January 6
}

\begin{abstract}
The mid-IR flux ratios $F_{A} / F_{B}=2.84 \pm 0.06$ of the two images of the gravitationally lensed quasar HE 11041805 show no wavelength dependence to within $3 \%$ across $3.6-8.0 \mu \mathrm{m}$, show no time dependence over 6 months, and agree with the broad emission-line flux ratios. This indicates that the mid-IR emission likely comes from scales large enough to be little affected by microlensing and that there is little differential extinction between the images. We measure a revised time delay between these two images of $152.2_{-3.0}^{+2.8}(1 \sigma)$ days from $R$ - and $V$-band data covering the years 1997-2006. This time delay indicates that the lens has an approximately flat rotation curve over scales of $1-2 R_{e}$. We also observed uncorrelated variations of $\sim 0.05 \mathrm{mag} \mathrm{yr}^{-1}$, which we attribute to microlensing of the optical emission from the accretion disk. The optical colors have also changed significantly in the sense that image $\mathrm{A}$ is now redder than image B, rather than bluer, as it was in 1993.
\end{abstract}

Subject headings: gravitational lensing — quasars: general — quasars: individual (HE 1104-1805)

Online material: machine-readable table

\section{INTRODUCTION}

The fluxes of gravitationally lensed images are affected by absorption in the interstellar medium (ISM; e.g., Falco et al. 1999) and magnification perturbations from satellites (substructure; Dalal \& Kochanek 2002) and stars (microlensing; for a review, see Wambsganss 2006). The simplest means of disentangling these effects is to explore the changes in the flux ratios with wavelength and time. In particular, the mid-IR flux ratios should be immune to microlensing because of the large size of the mid-IR emission region (e.g., Barvainis 1987) and insensitive to dust extinction because of the long wavelength. Broad emission line regions should also be insensitive to microlensing because of their large size (but see Richards et al. 2004), but may require corrections for extinction (e.g., Muñoz et al. 2004). Neither the mid-IR nor the broad-line flux ratios are immune to magnification perturbations from substructure. Unfortunately, it is difficult to measure mid-IR flux ratios of lenses because of the low sensitivity of even the best ground-based telescopes and the low resolution of current space-based mid-IR telescopes. Nonetheless, slow progress has been made with the measurement of mid-IR flux ratios for QSO 2237+0305 (Agol et al. 2000) and for PG 1115+080 and B1422+231 (Chiba et al. 2005). Since the mid-IR probably provides the best means of isolating the effects of substructure from those of microlensing and the ISM, we have tried to obtain Spitzer Infrared Array Camera mid-IR observations, when feasible, to resolve the lens system and measure the flux ratios.

Time delay measurements have traditionally been pursued for determining the Hubble constant $\left(H_{0}\right)$ independently of local distance indicators (Refsdal 1964). Alternatively, one can assume a value of $H_{0}$ and use the time delay information to learn about the mass profile of the lens (e.g., Kochanek 2002). Time delay measurements are also important for distinguishing intrinsic source variability from microlensing variability caused by stars in the

\footnotetext{
${ }^{1}$ Department of Astronomy, Ohio State University, Columbus, OH 43210; sdp@astronomy.ohio-state.edu,nmorgan@astronomy.ohio-state.edu, ckochanek@ astronomy.ohio-state.edu.

${ }_{2}^{2}$ Harvard-Smithsonian Center for Astrophysics, Cambridge, MA 02138.
}

lens galaxy. Once the two phenomena are separated, analysis of the microlensing variability can yield estimates of the mean stellar mass and the stellar surface density of the lens galaxy and can be used to measure the size of the source quasar (e.g., Kochanek et al. 2007). The level of microlensing variability in the optical is thus complementary to that in the mid-IR as a probe of magnification perturbations in gravitational lenses.

Here we report the results of new observations of HE 11041805 , a doubly imaged radio-quiet quasar at $z_{s}=2.319$, with a separation of 3.15", from the Hamburg/ESO survey (Wisotzki et al. 1993). The lens at $z_{l}=0.729$ was discovered in the nearIR by Courbin et al. (1998) and has been observed with the Hubble Space Telescope (HST; Remy et al. 1998; Lehár et al. 2000). After a series of initial attempts at measuring a time delay (Wisotzki et al. 1998; Gil-Merino et al. 2002; Schechter et al. 2003), Ofek \& Maoz (2003) succeeded, with an estimate of $161_{-711}^{+7,+34}(1 \sigma, 2 \sigma)$ days that was confirmed by Wyrzykowski et al. (2003). One source of difficulty is that the lens suffers considerably from microlensing effects. In particular, the flux ratios of the broad emission lines and the continuum are dramatically different (Wisotzki et al. 1993), the continuum flux ratio varies with wavelength (Wisotzki et al. 1998), and Schechter et al. (2003) found uncorrelated short-timescale variability between the images at the level of $0.06 \mathrm{mag}$.

In this paper we present new photometric data for HE 11041805 from Spitzer, the Small and Moderate Aperture Research Telescope System (SMARTS), and the Southern Astrophysical Research (SOAR) telescope in $\S 2$. We combine our new $R$-band data with the data from Schechter et al. (2003), Ofek \& Maoz (2003), and Wyrzykowski et al. (2003) to make an improved time delay estimate for HE 1104-1805 in $\S 3$, and we interpret its consequences for the lens model in $\S 4$. We summarize our results in $\S 5$.

\section{DATA}

In this section we discuss our mid-IR, near-IR, and optical observations. The mid-IR photometry is presented in Table 1, and the optical and near-IR photometry is presented in Table 3. 
TABLE 1

Spitzer IRAC OBSERVATIONS OF HE 1104-1805

\begin{tabular}{|c|c|c|c|c|c|}
\hline HJD & $\begin{array}{c}\lambda \\
(\mu \mathrm{m})\end{array}$ & $\mathrm{A} / \mathrm{B}$ & A & B & G \\
\hline \multirow[t]{4}{*}{3536.73 .. } & 3.6 & $2.87 \pm 0.09$ & $22.82 \pm 0.04$ & $23.96 \pm 0.02$ & $24.6 \pm 0.2$ \\
\hline & 4.5 & $2.82 \pm 0.08$ & $22.09 \pm 0.05$ & $23.46 \pm 0.04$ & $24.2 \pm 0.4$ \\
\hline & 5.8 & $2.84 \pm 0.06$ & $20.92 \pm 0.02$ & $22.05 \pm 0.02$ & $25.0 \pm 1.2$ \\
\hline & 8.0 & $2.90 \pm 0.03$ & $19.64 \pm 0.02$ & $20.79 \pm 0.01$ & $26.0 \pm 5.3$ \\
\hline \multirow[t]{4}{*}{3737.85.} & 3.6 & $2.88 \pm 0.09$ & $22.82 \pm 0.04$ & $23.97 \pm 0.02$ & $24.5 \pm 0.2$ \\
\hline & 4.5 & $2.83 \pm 0.08$ & $22.06 \pm 0.05$ & $23.19 \pm 0.03$ & $25.1 \pm 0.8$ \\
\hline & 5.8 & $2.73 \pm 0.06$ & $20.95 \pm 0.02$ & $22.04 \pm 0.02$ & $24.8 \pm 1.0$ \\
\hline & 8.0 & $2.78 \pm 0.03$ & $19.68 \pm 0.02$ & $20.79 \pm 0.01$ & $>24.2$ \\
\hline
\end{tabular}

Notes.-HJD is the heliocentric Julian date minus 2,450,000. The $\lambda$ column gives the wavelength in units of $\mu \mathrm{m}$. The A/B column gives the flux ratio of image A to image $\mathrm{B}$. The A, B, and $\mathrm{G}$ columns give the measured Vega magnitudes of image A, image B, and the lensing galaxy, respectively, with errors estimated from the bootstrap resampling technique. In the second-epoch $8 \mu \mathrm{m}$ observation, we present an upper limit from the bootstrap sample, since our best-fit model assigned it a slightly negative flux.

Each of these observations makes use of the HST astrometry for the lens and image positions and HST photometry for the lens galaxy profile.

\subsection{HST Observations}

HE 1104-1805 was originally observed with HST by Lehár et al. (2000), but we obtained deeper NICMOS NIC2 F160W ( $H$ band) observations as part of GO-9375 on 2003 December 17 (HJD 2,452,990.9), with the aim of improving the lens galaxy astrometry and profile and studying the quasar host galaxy. These covered five orbits and included observations of a nearby pointspread function (PSF) template star. The observations of the lens consisted of 22 dithered subexposures with 17, 1, 3, and 1 subexposures, having integration times of $640,448,128$, and $64 \mathrm{~s}$, respectively, for a total exposure time of $3.27 \mathrm{hr}$. The short exposures were simply used to fill orbits, given the timing restrictions of the long exposures. The images were analyzed as in Lehár et al. (2000). While the relative astrometry of the quasar images is identical to the single-orbit results in Lehár et al. (2000), the new estimate of the position of the lens galaxy relative to image A $(\Delta$ R.A. $=0.965 \pm 0.003, \Delta$ decl. $=-0.500 \pm 0.003)$ is shifted by approximately $0.01^{\prime \prime}(2 \sigma)$ in both coordinates. The quasar magnitudes are $15.57 \pm 0.03$ and $17.04 \pm 0.03$ for images $A$ and $B$, respectively. The lens galaxy is estimated to have a major axis effective radius of $R_{e}=0.72^{\prime \prime} \pm 0.07^{\prime \prime}$, an axis ratio of $q=0.80 \pm$ 0.01 , a major axis position angle of $48^{\circ} \pm 4^{\circ}$, and a total magnitude of $H=17.52 \pm 0.09 \mathrm{mag}$. These parameters for the lens galaxy are all consistent with the earlier results in Lehár et al. (2000).

\subsection{Spitzer IRAC Observations}

We observed HE 1104-1805 with Spitzer and the Infrared Array Camera (IRAC; Fazio et al. 2004) on 2005 June 15 and 2006 January 2 as part of Spitzer program 20451. Each observation consisted of 36 dithered $10.4 \mathrm{~s}$ images in each of the four IRAC channels: $3.6 \mu \mathrm{m}, 4.5 \mu \mathrm{m}, 5.8 \mu \mathrm{m}$, and $8.0 \mu \mathrm{m}$. The FWHM of the PSF at $3.6 \mu \mathrm{m}$ is $\sim 1.5^{\prime \prime}$, so we can easily resolve the QSO images, but we must worry about the flux of the lens galaxy, which is only $1.0^{\prime \prime}$ from the brighter QSO image. The spectral energy distribution of the lens galaxy will peak near $2.7 \mu \mathrm{m}$ and then steadily drop as we progress through the IRAC bands. Thus, as the resolution of the observations diminishes, the emission from the galaxy also becomes less important. We drizzled the images, using the MOPEX package, ${ }^{3}$ to a $0.3^{\prime \prime}$ pixel $^{-1}$

\footnotetext{
3 Available at http://ssc.spitzer.caltech.edu/postbcd/.
}

scale. To reduce the number of free parameters, we fix the relative positions of the QSO images and the lens galaxy, as well as the structure of the lens galaxy, using the HST observations mentioned in $\S 2.1$. We used the methods of Lehár et al. (2000) and the point response functions (PRFs) from the MOPEX package to measure the fluxes of the three components. The PRFs are 2 times oversampled relative to our drizzled images, so we binned them to match our images.

We used a bootstrap resampling method to estimate the errors in the fluxes and flux ratios. For each trial we randomly chose 36 images with replacement from the 36 subimages, drizzled these images together, and then fitted this synthetic image to estimate the component fluxes. We repeated this 200 times for each of the IRAC bands, and then we used the standard deviation of the flux ratios measured from the synthetic images as an estimate of the errors on the measured flux ratios. This process should naturally include both Poisson and systematic errors due to image pixel sampling in our uncertainties. The errors for the individual image magnitudes and the lens galaxy magnitude were estimated in the same way. The quasar flux ratios are the same in both epochs and in all four IRAC channels (see Table 1), and they agree with the emission-line flux ratios of 2.8 (with no error given) measured by Wisotzki et al. (1993). The galaxy magnitudes are given in Table 1, but the uncertainties are too large to measure the colors accurately because of flux tradeoffs with the brighter quasar image. On the basis of the lens redshift, an early-type galaxy should have IRAC colors of [3.6] $-[4.5]=$ -0.05 and $[5.8]-[8.0]=-0.03$ (R. Assef 2006, private communication). Our measurements are consistent with these colors, with large uncertainties.

\subsection{Monitoring Data}

Most of our optical and near-IR data were obtained using the queue-scheduled SMARTS $1.3 \mathrm{~m}$ telescope with the ANDICAM optical/infrared camera (DePoy et al. 2003). Between 2003 December 3 and 2006 May 22, we obtained 97 epochs in our primary monitoring band, the $R$ band, as well as sparse observations in the $B$ and $I$ bands. A simultaneous $J$-band image is obtained at all epochs. We used the method of Kochanek et al. (2006) to obtain the relative photometry of both images. For each band we determined the flux of the lens galaxy by fitting the images as a function of galaxy flux and then adopted the flux that optimized the goodness of fit for all images simultaneously. For calibration in future epochs, we list the positions and relative fluxes for our field reference stars in Table 2 . The relative 
TABLE 2

Relative Astrometry and Photometry for Field Reference Stars

\begin{tabular}{|c|c|c|c|c|}
\hline ID & $\begin{array}{l}\Delta \text { R.A. } \\
(\operatorname{arcsec})\end{array}$ & $\begin{array}{l}\Delta \text { decl. } \\
(\operatorname{arcsec})\end{array}$ & $\begin{array}{c}R \text {-Band } \Delta m \\
(\mathrm{mag})\end{array}$ & $\begin{array}{c}J \text {-Band } \Delta m \\
(\mathrm{mag})\end{array}$ \\
\hline $1 \ldots \ldots \ldots \ldots \ldots \ldots \ldots$ & -3.4 & -15.1 & $\equiv 0$ & $\equiv 0$ \\
\hline $2 \ldots \ldots \ldots \ldots \ldots \ldots \ldots$ & 31.8 & -5.3 & $0.731 \pm 0.002$ & $\ldots$ \\
\hline 3.................... & -45.7 & 23.8 & $0.604 \pm 0.002$ & $0.653 \pm 0.009$ \\
\hline $4 \ldots \ldots \ldots$ & 33.6 & 119.3 & $-0.003 \pm 0.003$ & $\ldots$ \\
\hline $5 \ldots \ldots \ldots \ldots \ldots \ldots \ldots \ldots$ & -143.9 & -64.4 & $-1.896 \pm 0.028$ & $\ldots$ \\
\hline
\end{tabular}

Notes.-The positions are measured with respect to HE 1104-1805 image A. The magnitude differences are for the SMARTS $R$ - and $J$-band data. For the SOAR data, only star 1 (set to unity) was available as a local reference star.

magnitudes for images $\mathrm{A}$ and $\mathrm{B}$ given in Table 3 are with respect to the first star in Table 2 .

\subsection{Near-IR SOAR Data}

We obtained a single epoch of near-IR data for HE 11041805 using the SOAR $4.1 \mathrm{~m}$ telescope at Cerro Pachon, Chile. Observations were taken on 2006 January 14 with OSIRIS (DePoy et al. 1993) operating at $\mathrm{f} / 7$, giving a plate scale of 0.139 arcsec pixel $^{-1}$ and a field of view of $2.4^{\prime}$ on a side. The exposures consisted of 16,18, and 17 box-dithered images through the $J, H$, and $K_{s}$ Barr filters, respectively, with exposure times of $10 \mathrm{~s}$ per image. Along with the $0.7^{\prime \prime}-0.75^{\prime \prime}$ FWHM seeing, this provided a sufficient signal-to-noise ratio in all filters to identify the two quasars and the lensing galaxy by eye after proper sky subtraction and image registration. Flux from image B was well isolated, but the 5 pixel seeing disk and the 8 pixel separation of image $\mathrm{A}$ from the lens galaxy meant that significant flux overlap between these two components was unavoidable. We used the same technique to optimize the galaxy flux as with the SMARTS data, namely, choosing the galaxy flux that gave the lowest $\chi^{2}$ residuals after again fixing the galaxy size to that measured from the HST data, which for this single epoch of data simply meant fitting all three component fluxes simultaneously, while again holding the relative positions fixed. As a check on the flux decomposition, the SOAR $J$-band A$\mathrm{B}$ magnitude difference of $-1.33 \pm 0.10 \mathrm{mag}$ is consistent with the SMARTS $J$-band value of $-1.39 \pm 0.03$ mag taken a night earlier. The fitting results for all three filters are given in Table 3.

\section{TIME DELAY ESTIMATES AND MICROLENSING}

For the time delay determination we combined the SMARTS $R$-band data with the Wise Observatory $R$-band data from Ofek \& Maoz (2003) and Optical Gravitational Lensing Experiment (OGLE) $V$-band data (Schechter et al. 2003; Wyrzykowski et al. 2003). The $V$-band data were adjusted to match the $R$-band data as in Ofek \& Maoz (2003). The resulting light curve is shown in Figure 1. Three Wise observations of image A (MJD epochs 2084.254, 1717.253, and 1557.520) were clearly outliers from the light curves, and they were masked in our time delay estimate. In total we used 408 epochs (or 816 data points) to estimate the time delay.

In the OGLE data, the A image shows rapid variability at the level of 0.06 mag on 1 month timescales that is not observed in image B, which Schechter et al. (2003) argued is due to the microlensing of hot spots in the accretion disk. In order to compensate for this systematic noise, we attempted to rescale the errors for each image and for each observatory in an unbiased way by fitting a Legendre polynomial with $N_{s}=40$ to each image separately with no time delay to estimate the factor by which the error estimates for each data set must be rescaled to be consistent with a smooth fitting curve. The factors for rescaling the Wise, OGLE, and SMARTS data for image A are 2.4, 4.6, and 1.0, respectively, and those for image B are 1.1, 1.2, and 0.9. Since using a polynomial order of $N_{s}=40$ for the error renormalization is somewhat arbitrary, we also tried $N_{s}=70$, and the final time delay estimate changed by only $\sim 1 \%$.

We determined the time delay using the polynomial fitting methods of Kochanek et al. (2006). We divided the data into two observing periods at $\mathrm{HJD}=2,452,708$ because of a considerable gap in the data. In each observing period we modeled the flux of the source by Legendre polynomials of order $N_{s}=20,30, \ldots$, 80 and the microlensing variability by Legendre polynomials of order $N_{\mu}=1,2, \ldots, 6$. Each model $m$ then leads to a $\chi^{2}$ fit statistic $\chi_{m}^{2}(\Delta t)$ that can be used to estimate the delay and whose value at the minimum relative to the number of degrees of freedom, $N_{\text {dof }}(m)$, can be used to evaluate the significance of the fit. One issue with the polynomial method is the choice of polynomial order. Our previous approach (Kochanek et al. 2006) used the $F$-test to determine when increasing the order of the fitting polynomials was statistically unnecessary. Using the same technique for HE 1104-1805, we find that orders more complex than $N_{s}=50$ and $N_{\mu}=4$ are not required by the data, and this model gives a time delay estimate of $157.2 \pm 2.6$ days.

TABLE 3

Light Curves For HE 1104-1805

\begin{tabular}{|c|c|c|c|c|c|}
\hline HJD & $\chi^{2} /$ dof & Comp. A & Comp. B & Ref. Stars & Telescope \\
\hline $2976.806 .$. & 0.16 & $0.118 \pm 0.031$ & $1.663 \pm 0.092$ & $0.024 \pm 0.009$ & SMARTS $J$ \\
\hline $2976.807 \ldots \ldots$. & 2.16 & $-0.012 \pm 0.010$ & $1.379 \pm 0.012$ & $0.043 \pm 0.005$ & SMARTS $R$ \\
\hline $2985.815 \ldots \ldots \ldots \ldots \ldots \ldots \ldots$ & 0.19 & $0.134 \pm 0.027$ & $1.575 \pm 0.073$ & $0.027 \pm 0.009$ & SMARTS $J$ \\
\hline $2985.818 \ldots \ldots \ldots \ldots \ldots \ldots$ & 1.53 & $-0.017 \pm 0.010$ & $1.390 \pm 0.013$ & $0.035 \pm 0.005$ & SMARTS $R$ \\
\hline $2993.822 \ldots \ldots \ldots \ldots \ldots \ldots \ldots$ & 0.10 & $0.114 \pm 0.029$ & $1.526 \pm 0.076$ & $0.024 \pm 0.009$ & SMARTS $J$ \\
\hline $2993.826 \ldots \ldots \ldots \ldots$ & 2.76 & $-0.028 \pm 0.010$ & $1.403 \pm 0.013$ & $0.034 \pm 0.005$ & SMARTS $R$ \\
\hline $3000.817 \ldots \ldots \ldots \ldots \ldots \ldots \ldots \ldots$ & 0.17 & $0.098 \pm 0.024$ & $1.630 \pm 0.063$ & $0.030 \pm 0.009$ & SMARTS $J$ \\
\hline $3000.820 \ldots \ldots \ldots \ldots \ldots \ldots \ldots \ldots$ & 3.95 & $-0.049 \pm 0.010$ & $1.405 \pm 0.012$ & $0.048 \pm 0.005$ & SMARTS $R$ \\
\hline $3009.812 \ldots \ldots \ldots$ & 0.15 & $0.061 \pm 0.023$ & $1.623 \pm 0.063$ & $0.028 \pm 0.009$ & SMARTS $J$ \\
\hline $3009.815 \ldots \ldots \ldots$ & 2.31 & $-0.087 \pm 0.010$ & $1.408 \pm 0.012$ & $0.040 \pm 0.005$ & SMARTS $R$ \\
\hline
\end{tabular}

Notes.- HJD is the heliocentric Julian date minus 2,450,000. The A and B columns give the image magnitudes relative to the local reference stars. The column labeled "Ref. Stars" gives the average magnitude differences of the reference stars relative to the campaign mean. Table 3 is presented in its entirety in the electronic edition of the Astrophysical Journal. A portion is shown here for guidance regarding its form and content. 


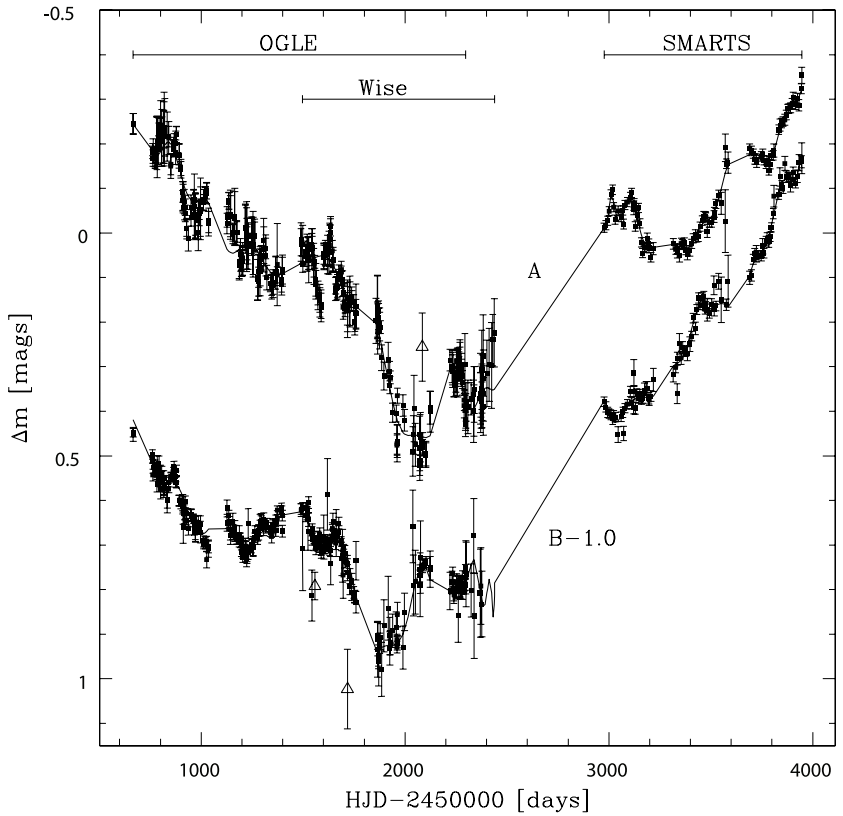

FIG. 1.-Light curve used for our time delay estimate. It includes only the SMARTS $R$-band, OGLE $V$-band, and Wise $R$-band data, where bars at the top indicate the period during which each observatory monitored the system. The three open symbols indicate Wise data points that were not considered in the final time delay estimate. The (lower) image B light curve has been shifted by 1 mag. The 15 points with errors greater than 0.1 mag have been suppressed for clarity.

A potential issue with using the $F$-test is that the restriction of the final estimate to a single model may underestimate the uncertainties by neglecting the effects of shifts in the delay due to changes in the source or microlensing model. We can use Bayesian methods to perform a reasonable average over the results for different models by adding an information criterion for the significance of changes in the numbers of model parameters. If model $m$ has $N_{m}$ parameters, then we assign it a likelihood of fitting the data of

$$
P(D \mid \Delta t, m) \propto \exp \left[-\chi_{m}^{2}(\Delta t) / 2-k N_{m}\right],
$$

so a model with more parameters needs to reduce $\chi_{m}^{2}$ by $2 k$ for each additional parameter to represent an improvement in the fit. We considered the two cases of $k=1$ (which corresponds to the Akaike information criterion [AIC]) and $k=\ln n$ (where $n$ equals the number of data; this corresponds to the Bayesian information criterion [BIC]). The AIC treats new parameters more favorably than the BIC. The best models using the AIC weights have $N_{s}=80$ and $N_{\mu}=5$, while those for the BIC weights have $N_{s}=30$ and $N_{\mu}=4$. The time delay estimate is then the average over all the models,

$$
P(\Delta t \mid D) \propto \sum_{m} P(D \mid \Delta t, m)
$$

where we assume a uniform prior for the different models after adding the information criteria. Thus, models of similar likelihoods contribute equally to the delay estimate, while models with low likelihoods contribute little.

Figure 2 shows the resulting probability distribution for both of these criteria and with the photometric uncertainties renormalized using either the $N_{s}=40$ or $N_{s}=70$ model for a smooth light curve. The result for these four cases (Table 4) are mutually consistent and are roughly consistent with our previous $F$-test approach. We adopt the broadest of these four Bayesian estimates, $\Delta t_{A B}=t_{A}-t_{B}=152.2_{-3.0}^{+2.8}(1 \sigma)$ days, in the sense that

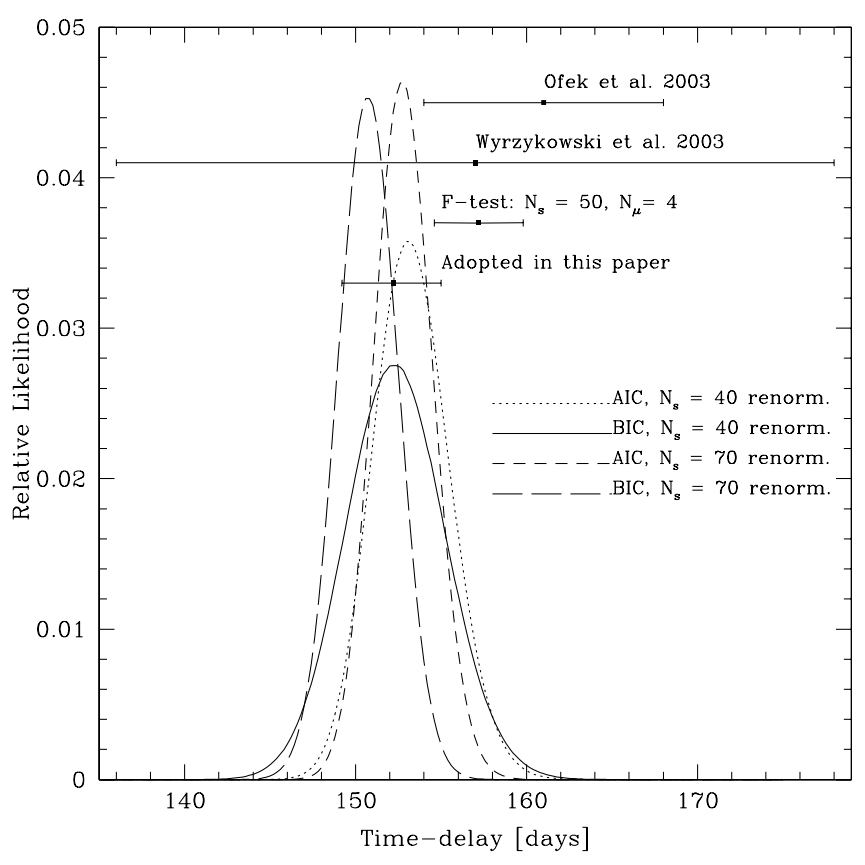

FIG. 2.-Relative likelihood for each time delay $\Delta t$ for the two different error renormalization schemes $\left(N_{s}=40\right.$ or $\left.N_{s}=70\right)$ and the two different information criteria (AIC and BIC). The points with error bars indicate our adopted delay estimates and the Ofek \& Maoz (2003; excluding systematic errors), OGLE, and $F$-test delay estimates. We adopt the broadest of the Bayesian estimates (BIC, with $N_{s}=40$ ) as our standard estimate.

image B leads image A, as our standard estimate. None of the Bayesian models gives significant weight to the model singled out by the $F$-test, although they are marginally consistent and the difference in the delay is only $3 \%$. The delay in the model favored by the $F$-test is curiously shifted from the time delay given by most models with comparable polynomial order. It also has a $\chi^{2}(\Delta t)$ minimum intermediate to those favored by both the $\mathrm{AIC}$ and the BIC, so it is given little weight in either case. The Bayesian estimates are consistent with the best previous measurements of $161_{-7,-11}^{+7,+34}(1 \sigma, 2 \sigma)$ days by Ofek \& Maoz (2003) and $157 \pm 21$ days by Wyrzykowski et al. (2003). These analysisdependent shifts suggest that the measurement uncertainties should be interpreted conservatively. Moreover, the formal $2 \%$ measurement errors are in the regime where the cosmic variance of order 5\% in the delays due to the structures along the line of sight is a significant source of uncertainty (e.g., Bar-Kana 1996).

Given the time delay, we can shift the light curves to obtain an estimate of the fluctuations due to microlensing, as shown in Figure 3 for our standard model. Over the decade since the discovery of HE 1104-1805, its flux ratio has been changing by

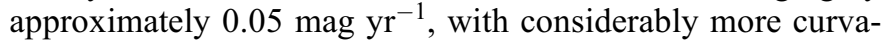
ture over the last few years, as the flux ratio approaches that

TABLE 4

\begin{tabular}{|c|c|c|}
\hline Criterion & Renormalization & Time Delay \\
\hline AIC... & $N_{s}=40$ & $153.0_{-2.2}^{+2.4}$ \\
\hline BIC ... & $N_{s}=40$ & $152.2_{-3.0}^{+2.8}$ \\
\hline AIC.... & $N_{s}=70$ & $152.6_{-1.8}^{+1.0}$ \\
\hline 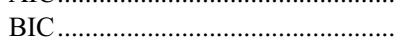 & $N_{s}=70$ & $150.6_{-1.8}^{+1.8}$ \\
\hline$F$-test ................. & $N_{s}=40$ & $157.2_{-2.6}^{+2.6}$ \\
\hline
\end{tabular}

Time Delay Estimates

Note.-The criterion column indicates the statistical method, and the renormalization column indicates the polynomial order used to renormalize the error bars (see $\S 3$ ). 


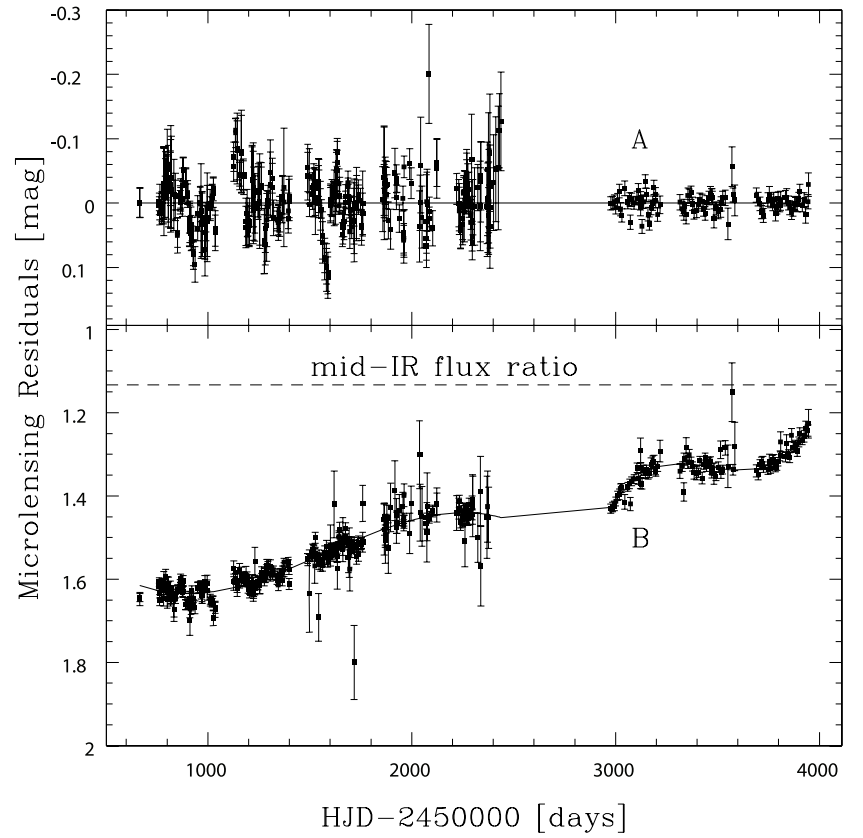

FIG. 3.-Microlensing variability in HE 1104-1805. The points show the data after subtracting the model for the source variability from our standard model, and the curves show the model for the microlensing variability. The fit is broken at $\mathrm{HJD}=2,452,708$ into two segments. By definition we assign the variability to image B, but we can only determine the difference between images A and $\mathrm{B}$. Note how the flux ratio has been steadily approaching that measured from the broad emission lines or at mid-IR wavelengths.

measured in the mid-IR or from the broad emission lines. The long time delay means that there is significant microlensing variability on the timescale of the delay, making it essential to explore a broad range of microlensing models when estimating the delay. Figure 4 shows the wavelength-dependent flux ratios of the images at the time of the Spitzer observations and when it was discovered (Gil-Merino et al. 2002; Remy et al. 1998). The optical A - B colors have become significantly redder and the flux ratio has become much smaller since the discovery of this system. There is also a curious reversal in the color trends near $1.2 \mu \mathrm{m}$.

\section{LENS MODELS}

We modeled the system by combining a constant $-M / L$ de Vaucouleurs model with a Navarro-Frenk-White (NFW) halo using the lensmodel program of the gravlens package (Keeton 2001). The constant- $M / L$ model represents the visible lens galaxy, which we model with an ellipticity of $e=1-q=0.20 \pm$ 0.04 , a position angle of P.A. $=48^{\circ} \pm 4^{\circ}$ (east through north), and an effective radius of $R_{e}=0.72^{\prime \prime}$, as determined from the HST observations discussed in $\S 2.1$. We broadened the uncertainties in the axis ratio from the fits in $\S 2.1$, since we also use this axis ratio and position angle for the ellipsoidal NFW component. We used a prior on the external shear of $\gamma=0.05 \pm 0.05$, and we assumed a cosmology with $\Omega_{m}=0.3, \Omega_{\Lambda}=0.7$, and $H_{0}=72 \pm$ $7 \mathrm{~km} \mathrm{~s}^{-1} \mathrm{Mpc}^{-1}$ (Freedman et al. 2001). We used a scale length of $r_{s}=10.0^{\prime \prime}$ for the NFW model. The results will depend only weakly on $r_{s}$ if it is significantly larger than the image radii. We constrained the flux ratios to $F_{A / B}=2.84 \pm 0.06$, the average of the mid-IR flux ratios. As a two-image lens with modest image magnifications (11.5 and 4.0), the HE 1104-1805 image fluxes should be relatively immune to perturbations from substructure (e.g., Mao \& Schneider 1998). We used our newly estimated time delay of $\Delta t_{A B}=t_{A}-t_{B}=152 \pm 9$ days, with the errors broadened to $6 \%$ to account for cosmic variance.

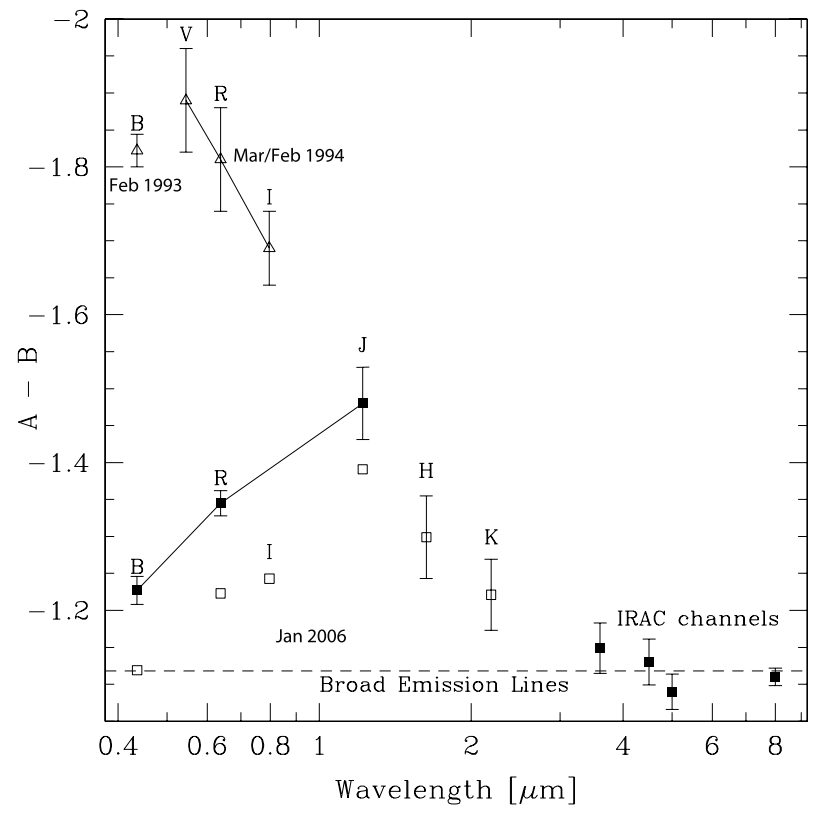

FIG. 4.-Flux ratio vs. wavelength. Solid (open) symbols represent flux ratios that are (are not) corrected for the time delay. Squares are used for the 2006 January data, while triangles are used for flux ratios from 1993 and 1994. Note that the recent flux ratios are shifted by about $0.1 \mathrm{mag}$ when we correct for the time delay due to the relatively rapid source variability. While we do not have time delay-corrected $H$ - and $K$-band flux ratios, we expect that they are affected less by the time delay, because the IRAC channel flux ratios did not change on the time delay scale. Note how the flux ratio is now much closer to the mid-IR and emission-line flux ratios and that the optical flux ratios now have image A redder than image B, while the reverse still holds in the near-IR.

Figure 5 shows the goodness of fit as a function of $f_{M / L}$, where $f_{M / L}$ is the fraction of mass in the de Vaucouleurs component compared to a constant $-M / L$ model in which the NFW component has no mass $\left(f_{M / L}=1\right)$. The best-fit model has $f_{M / L}=0.30_{-0.05}^{+0.04}$. Figure 6 shows the monopole deflection profile of these models, which is similar to the square of the rotation curve. We see that

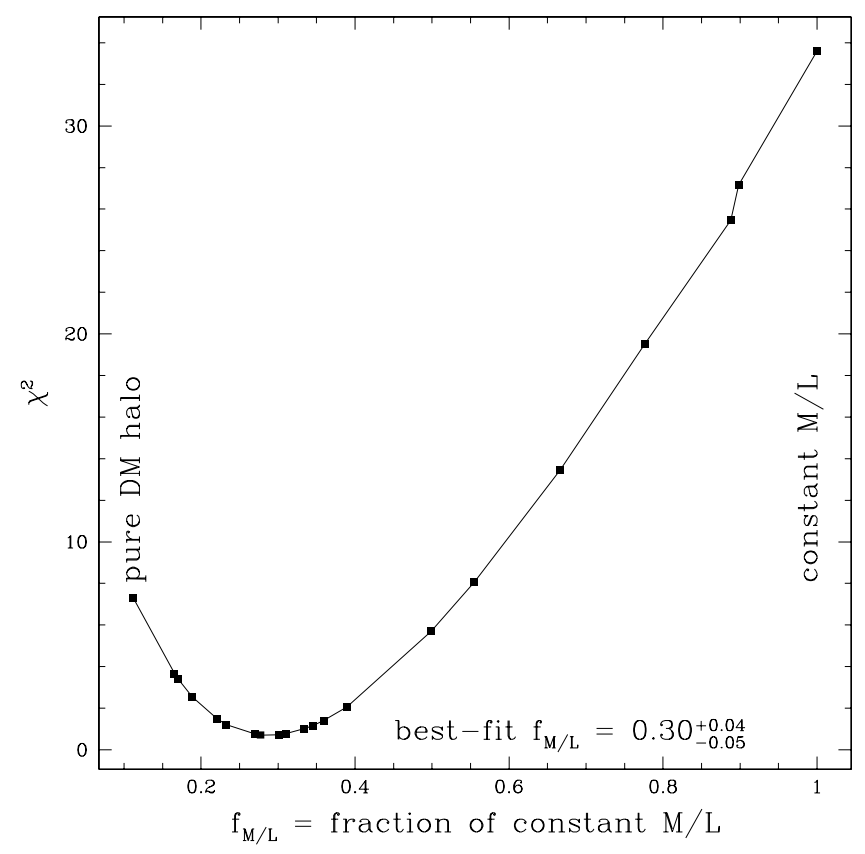

FIG. 5.- Goodness of fit for the "galaxy plus halo" models. The $\chi^{2}$ is plotted as a function of $f_{M / L}$, which is the mass of the de Vaucouleurs component divided by the mass of the best-fitting constant- $M / L$ de Vaucouleurs model. 


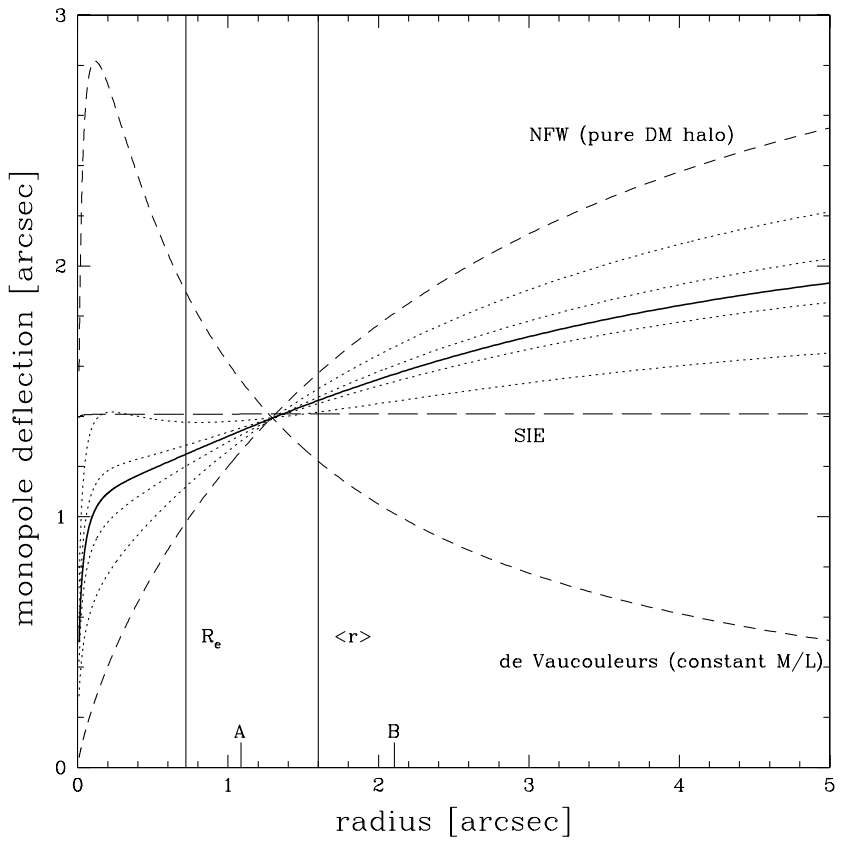

FIG. 6.-Radial deflection profiles, which roughly correspond to the square of the rotation curve, for the de Vaucouleurs plus NFW models. The solid curve shows the best-fit model with $f_{M / L}=0.30$. The four dotted curves show the $1 \sigma$ and $2 \sigma$ bounds to the best-fit model. The dashed lines show the profiles for the pure NFW model, a singular isothermal ellipsoid (SIE) model, and a constant$M / L$ model. The vertical lines mark the effective radius, $R_{e}$, and the mean radius of the images, $\langle r\rangle$. The radial distances of the images from the center of the galaxy are labeled "A" and "B."

the best-fit models have quasi-flat rotation curves on scales of $1-2 R_{e}$. In fact, a model using a singular isothermal ellipsoid fits the data reasonably well, but is formally ruled out at approximately $95 \%$ confidence $\left(\Delta \chi^{2}=3.6\right)$.

\section{CONCLUSIONS}

We find that the mid-IR flux ratios of $F_{A} / F_{B}=2.84 \pm 0.06$ in HE 1104-1805 agree with the broad emission line flux ratios of 2.8 (Wisotzki et al. 1993). The flux ratios from 3.6 to $8.0 \mu \mathrm{m}$ did not change over the 6 month period between the two IRAC epochs. The optical flux ratios have fallen from 4.6, when the lens was discovered, to a value of 3.1 today that approaches the ratio in the mid-IR and emission lines, indicating that there is significant microlensing of the quasar accretion disk. This means, as expected, that the mid-IR/broad line emission regions are large compared to the Einstein radius of the microlenses, $R_{\mathrm{E}}=(3.6 \times$ $\left.10^{16} \mathrm{~cm}\right)\left(\langle M\rangle / h M_{\odot}\right)^{1 / 2}$, while the optical emission regions are significantly more compact. The microlensing is clearly chromatic, as image $A$ is now significantly redder relative to image $B$ than when the system was discovered in 1993. It also seems that as image A has faded relative to image $\mathrm{B}$, the excess variability in image $\mathrm{A}$ observed by Schechter et al. (2003) has vanished. We will explore this quantitatively in S. Poindexter et al. (2007, in preparation).

We have also determined a new time delay for HE 11041805, $\Delta t=t_{A}-t_{B}=152.2_{-3.0}^{+2.8}(1 \sigma)$ days, in the sense that image $B$ leads image A. This uncertainty should be interpreted conservatively, because there are some variations in the estimate with the analysis method and because cosmic variance is a significant contribution to the uncertainties when the delay is used to model the mass distribution of the lens. If we model the lens galaxy mass distribution as the observed de Vaucouleurs profile embedded in an NFW halo, we find the best-fit lens galaxy model has a stellar mass fraction in the de Vaucouleurs component that is $f_{M / L}=0.30_{-0.05}^{+0.04}$ of a constant- $M / L$ model. This ratio corresponds to a deflection profile that is marginally consistent $\left(\Delta \chi^{2}=3.56\right)$ with the system having a flat rotation curve. Thus, HE 1104-1805 has a mass distribution typical of gravitational lens galaxies (Rusin \& Kochanek 2005; Treu et al. 2006).

This work is based in part on observations made with the Spitzer Space Telescope, which is operated by the Jet Propulsion Laboratory, California Institute of Technology, under a contract with NASA. Support for this work was provided by NASA through an award GRT00003172 issued by JPL/Caltech. Based on observations made with the NASA/ESA Hubble Space Telescope, obtained at the Space Telescope Institute. STScI is operated by the association of Universities for Research in Astronomy, Inc., under NASA contract NAS 5-26555. OSIRIS is a collaborative project between the Ohio State University and Cerro Tololo Inter-American Observatory (CTIO) and was developed through NSF grants AST 90-16112 and AST 92-18449. CTIO is part of the National Optical Astronomy Observatory (NOAO), based in La Serena, Chile. NOAO is operated by the Association of Universities for Research in Astronomy (AURA), Inc., under cooperative agreement with the National Science Foundation. We would like to thank Roberto Assef for providing estimates of the IRAC colors for the lens galaxy.
Agol, E., Jones, B., \& Blaes, O. 2000, ApJ, 545, 657

Bar-Kana, R. 1996, ApJ, 468, 17

Barvainis, R. 1987, ApJ, 320, 537

Chiba, M., Minezaki, T., Kashikawa, N., Kataza, H., \& Inoue, K. T. 2005, ApJ, 627,53

Courbin, F., Lidman, C., \& Magain, P. 1998, A\&A, 330, 57

Dalal, N., \& Kochanek, C. S. 2002, ApJ, 572, 25

DePoy, D. L., Atwood, B., Byard, P. L., Frogel, J., \& O’Brien, T. P. 1993, Proc. SPIE, 1946, 667

DePoy, D. L., et al. 2003, Proc. SPIE, 4841, 827

Falco, E. E., et al. 1999, ApJ, 523, 617

Fazio, G. G., et al. 2004, ApJS, 154, 10

Freedman, W. L., et al. 2001, ApJ, 553, 47

Gil-Merino, R., Wisotzki, L., \& Wambsganss, J. 2002, A\&A, 381, 428

Keeton, C. R. 2001, preprint (astro-ph/0102340)

Kochanek, C. S. 2002, ApJ, 578, 25

Kochanek, C. S., Dai, X., Morgan, C., Morgan, N., Poindexter, S., \& Chartas, G. 2007, in Statistical Challenges in Modern Astronomy IV, ed. G. J. Babu \& E. D. Feigelson (San Francisco: ASP), in press (astro-ph/0609112)

Kochanek, C. S., Morgan, N. D., Falco, E. E., McLeod, B. A., Winn, J. N., Dembicky, J., \& Ketzeback, B. 2006, ApJ, 640, 47

\section{REFERENCES}

Lehár, J., et al. 2000, ApJ, 536, 584

Mao, S., \& Schneider, P. 1998, MNRAS, 295, 587

Muñoz, J. A., Falco, E. E., Kochanek, C. S., McLeod, B. A., \& Mediavilla, E. 2004, ApJ, 605, 614

Ofek, E. O., \& Maoz, D. 2003, ApJ, 594, 101

Refsdal, S. 1964, MNRAS, 128, 307

Remy, M., Claeskens, J.-F., Surdej, J., Hjorth, J., Refsdal, S., Wucknitz, O., Sørensen, A. N., \& Grundahl, F. 1998, NewA, 3, 379

Richards, G. T., et al. 2004, ApJ, 610, 679

Rusin, D., \& Kochanek, C. S. 2005, ApJ, 623, 666

Schechter, P. L., et al. 2003, ApJ, 584, 657

Treu, T., Koopmans, L. V., Bolton, A. S., Burles, S., \& Moustakas, L. A. 2006, ApJ, 640, 662

Wambsganss, J. 2006, in Gravitational Lensing: Strong, Weak and Micro, ed. G. Meylan, P. North, \& P. Jetzer (Berlin: Springer), 453

Wisotzki, L., Köhler, R., Kayser, R., \& Reimers, D. 1993, A\&A, 278, L15

Wisotzki, L., Wucknitz, O., Lopez, S., \& Sørensen, A. N. 1998, A\&A, 339, L73

Wyrzykowski, Ł., et al. 2003, Acta Astron., 53, 229 


\title{
ERRATUM: “MID-IR OBSERVATIONS AND A REVISED TIME DELAY FOR THE GRAVITATIONAL LENS SYSTEM QUASAR HE 1104-1805” (ApJ, 660, 146 [2007])
}

\author{
Shawn Poindexter, Nicholas Morgan, Christopher S. Kochanek, and Emilio E. Falco
}

The data in this erratum replace Table 1 . The Spitzer IRAC magnitudes were offset by +8.79 mag.

TABLE 1

Spitzer IRAC OBSERVATIONS OF HE 1104-1805

\begin{tabular}{|c|c|c|c|c|c|}
\hline HJD & $\begin{array}{c}\lambda \\
(\mu \mathrm{m})\end{array}$ & $\mathrm{A} / \mathrm{B}$ & A & B & G \\
\hline \multirow{4}{*}{$3536.73 \ldots \ldots \ldots \ldots \ldots \ldots \ldots \ldots \ldots \ldots$} & 3.6 & $2.87 \pm 0.09$ & $14.03 \pm 0.04$ & $15.17 \pm 0.02$ & $15.8 \pm 0.2$ \\
\hline & 4.5 & $2.82 \pm 0.08$ & $13.30 \pm 0.05$ & $14.67 \pm 0.04$ & $15.4 \pm 0.4$ \\
\hline & 5.8 & $2.84 \pm 0.06$ & $12.13 \pm 0.02$ & $13.26 \pm 0.02$ & $16.2 \pm 1.2$ \\
\hline & 8.0 & $2.90 \pm 0.03$ & $10.85 \pm 0.02$ & $12.00 \pm 0.01$ & $17.2 \pm 5.3$ \\
\hline \multirow[t]{4}{*}{$3737.85 \ldots \ldots \ldots \ldots \ldots \ldots \ldots$} & 3.6 & $2.88 \pm 0.09$ & $14.03 \pm 0.04$ & $15.18 \pm 0.02$ & $15.7 \pm 0.2$ \\
\hline & 4.5 & $2.83 \pm 0.08$ & $13.27 \pm 0.05$ & $14.40 \pm 0.03$ & $16.3 \pm 0.8$ \\
\hline & 5.8 & $2.73 \pm 0.06$ & $12.16 \pm 0.02$ & $13.25 \pm 0.02$ & $16.0 \pm 1.0$ \\
\hline & 8.0 & $2.78 \pm 0.03$ & $10.89 \pm 0.02$ & $12.00 \pm 0.01$ & $>15.4$ \\
\hline
\end{tabular}

Note.-HJD is the Heliocentric Julian minus 2,450,000. The $\lambda$ column is the wavelength in $\mu \mathrm{m}$. The A/B column is the flux ratio of image A to image B. The A, B, and $\mathrm{G}$ columns give the measured Vega magnitudes of image A, image B, and the lensing galaxy, respectively, with the errors estimated from the bootstrap resampling technique. In the secondepoch $8 \mu \mathrm{m}$ observation, we present an upper limit from the bootstrap sample, since our best-fit model assigned it a slightly negative flux. 